Stoa

Vol. 3, no. 5, 2012, pp. 37-63

ISSN 2007-1868

\title{
TÓPICOS KANTIANOS EN LA CONCEPCIÓN ESTRUCTURALISTA DE LAS TEORÍAS CIENTÍFICAS
}

\author{
Juan Manuel Jaramillo Uribe \\ Universidad del Valle \\ jaramillo.juanmanuel@gmail.com
}

RESUMEN: Este texto busca establecer la presencia de algunas tesis y nociones kantianas en el programa estructuralista de reconstrucción de teorías iniciado por J.D. Sneed y, en especial, mostrar cómo dicho programa hace realidad el ideal arquitectónico planteado por I. Kant en su Crítica de la Razón Pura. Aunque en la obra fundacional del programa, The Logical Structure of Mathematical Physics (1971), no hay ninguna referencia explícita a I. Kant, en Theorienstrukturen und Theoriendynamik de W. Stegmüller hay dos alusiones explícitas. La primera tiene que ver con la interpretación que Stegmüller hace de la noción de "presuposición" utilizada por Sneed al formular su criterio de teoricidad; la segunda, con la relación analógica entre el componente formal $K$ de las teorías empíricas de que habla el estructuralismo y el sistema de categorías kantiano. Finalmente, se abordará el problema realismo-antirrealismo en el marco de estas dos propuestas.

PALABRAS ClAVE: Kant - Sneed · estructuralismo $\cdot$ arquitectónica $\cdot$ presuposición

ABST RACT: The purpose of this paper is to establish the presence of some Kantian thesis and notions in the structuralist program of theory reconstruction initiated by J. D. Sneed. Especially, it shows how such a program realizes the ideal architectonic suggested by I. Kant his Critique of Pure Reason. Although, in the seminal work of the program, The Logical Structure of Mathematical Physics (1971), there is no explicit reference to I. Kant, in Theorienstrukturen und Theoriendynamik (1973) of W. Stegmüller there are two explicit mentions. The first one has to do with the interpretation Stegmüller suggests of the notion of "presupposition" used by Sneed when he formulates his criterion of theoricity; the second one, with the analogical relationship between the formal component $K$ of empirical theories discussed by the structuralism and the system of Kantian categories. Finally, it will be considered the problem of realismantirealism within the framework of these two proposals. 
KEYWORDS: Kant $\cdot$ Sneed $\cdot$ structuralism $\cdot$ architectonic $\cdot$ presupposition

\section{Introducción}

Para comenzar, considero necesario hacer algunas precisiones. En primer lugar, no se trata de presentar la concepción estructuralista de las teorías científicas como una forma de kantismo, o, para ser más exactos, de neokantismo, sino de establecer la presencia de algunas de las tesis kantianas en dicha concepción y, de modo especial, la manera en que el programa metateórico de reconstrucción estructuralista de las teorías científicas hace realidad el ideal arquitectónico esbozado por el filósofo königsberguense en su Crítica de la razón pura.

En segundo lugar, aunque hablamos de "la concepción estructuralista" esto no significa que entre sus miembros exista una concepción unánime, sino, más bien, una serie de propuestas específicas que, en general, comparten una estrategia general de análisis y una forma particular de presentar e identificar las teorías científicas: la forma modelística, a la que se incorporan de modo sistemático aportes importantes de algunos autores historicistas como Kuhn y Lakatos (Véase Moulines 2006, pp. 133-134).

Para disipar cualquier duda sobre un supuesto neokantismo en la concepción estructural, basta constatar, como hecho empírico, la ausencia de una referencia explícita a la obra del filósofo königsberguense en el libro fundacional de esta concepción: The Logical Structure of Mathematical Physics (1971) de J.D. Sneed; si bien, dos años después, en la presentación que de este libro hace W. Stegmüller en el segundo tomo su Theorie und Erfahrung titulado Theorienstrukturen und Theoriendynamik (1973) el autor hace dos referencias explícitas a Kant que, además de permitir clarificar aspectos importantes del programa estructuralista, se revisten de particular importancia por ser Stegmüller uno de los principales exégetas y críticos de la obra de Kant.

En la primera referencia, Stegmüller propone interpretar la noción de "presuposición" de que se vale Sneed en la formulación de su criterio de $T$-teoricidad como condiciones de posibilidad analíticotrascendentales en el sentido de Kant. En la segunda, plantea una analogía entre el componente formal de las teorías empíricas, a saber, su núcleo formal $K$, y el sistema de los conceptos a priori (categorías) del entendimiento a que alude Kant en su teoría del conoci- 
miento, pues tanto el núcleo estructural $K$ como dicho sistema son inmunes a los cambios en el campo de la experiencia; sin embargo, Stegmüller, frente a la rigidez de la concepción apriorística kantiana, propone una relativización temporal de dicho a priori que lo acerca a la interpretación neo-kantiana realizada por Cassirer. Aunque en este trabajo nos referiremos in extenso a estos dos tópicos kantianos de la "concepción estructural" destacados por Stegmüller, también mostraremos las posibles analogías entre la concepción kantiana y la concepción estructuralista con respecto al modo en que cada una de ellas se plantea la discusión de lo que Moulines ha denominado los aspectos onto-epistemo-semánticos de las teorías empíricas, y donde la discusión realismo-antirrealismo cobra particular importancia.

\section{Arquitectónica kantiana y arquitectónica estructuralista}

Desde la antigüedad clásica hasta nuestros días la mayor parte de los teóricos del conocimiento abrazaron el ideal de que éste tuviera un desarrollo arquitectónico y se organizara de tal manera que sus componentes formaran un todo articulado e integrado. Sin embargo, aunque el ideal de "sistematización del conocimiento" es bastante remoto, la noción de "sistema" como cuerpo de conocimiento orgánicamente estructurado sólo aparece en el Renacimiento tardío, pues para los antiguos dicha noción fundamentalmente dice relación a la organización del mundo físico y, por tanto, tiene una connotación eminentemente ontológica y no epistemológica -como lo muestra con especial lucidez N. Rescher en su libro Cognitive Systematization (1979). Años más tarde, Christian Wolf compendió en la siguiente definición: "systema est veritatum inter se et cum principiis suis connexarum congeries" "sistema es una colección de verdades debidamente ordenadas de acuerdo con los principios que gobiernan sus conexiones"), la cual constituyó un cambio verdaderamente significativo, pues con ella se introduce una noción epistémica de "sistema" que contrasta significativamente con la noción ontológica que hasta ese momento era dominante. Sin embargo, la teorización explícita de esta noción en su nuevo sentido epistémico sólo será realizada a mediados del siglo xviı por dos alemanes coetáneos: J.H. Lambert e I. Kant, quienes recogieron los resultados de la sistematización práctica realizada por filósofos y científicos 
como Descartes, Spinoza, Newton, Leibniz y Wolff. Al respecto Kant escribió:

Por una arquitectónica yo entiendo el arte de construir sistemas. Como la unidad sistemática es lo que primero eleva el conocimiento ordinario al rango de ciencia, esto es, lo transforma de un mero agregado de conocimientos en un sistema, la arquitectónica es la doctrina de lo científico en nuestro conocimiento, y, en consecuencia, necesariamente pertenece a la doctrina del método. [... ] Por sistema entiendo la unidad de los múltiples modos de conocimiento bajo una idea. Esta idea es el concepto proporcionado por la razón - de la forma de un todo- en cuanto que mediante tal concepto se determina a priori no sólo el alcance de su contenido múltiple, sino también la posición que ocupan las partes, unas con respeto a otras (Kant 1781/1787, A 832 /B 860).

Como se puede ver, para este filósofo la arquitectónica, como "el arte de construir sistemas", constituye un ideal para todo conocimiento que aspire a presentarse como ciencia, incluyendo -en su caso- el conocimiento filosófico que, como metafísica -tanto de la naturaleza como de la moral- no es otra cosa que el sistema de los conocimientos a priori. Pero la realización de este ideal tardará años, pues la Crítica de la razón pura -como lo reconoce su autor- es únicamente una preparación o propedéutica para, al menos, un canon de la razón, i.e., para la presentación de los principios a priori que, como fundamento, hacen posible el uso correcto de nuestra facultad cognoscitiva y el ordenamiento sistemático, i.e., arquitectónico, de los productos de esa facultad. En buena medida, toda la teoría del conocimiento del siglo xIx permanecerá atada a aquella propedéutica kantiana de justificar la posibilidad legisladora de la razón sobre la naturaleza, tomando como base principios que no provienen de ella. A este respecto cabe destacar las contribuciones de Hertz, Mach, Poincaré y Kirchhoff, cuya influencia será decisiva para la realización del primer gran proyecto arquitectónico en filosofía de la ciencia: el proyecto reconstruccionista del positivismo lógico y de lo que Putnam, en 1962, denominó la "Concepción Heredada".

En efecto, para los representantes del Círculo de Viena y para sus "herederos", la arquitectónica, como el arte de los sistemas, tendrá como método el análisis y reconstrucción lógicos de los aspectos sintácticos y, en menor medida, semánticos, de las teorías empíricas, y como instrumento el cálculo de predicados de primer orden con identidad. 
Consecuentemente, las teorías empíricas se caracterizaron como cálculos axiomáticos formulados en un lenguaje formal $L$ e interpretados a través de reglas semánticas $R$; es el caso de las llamadas "reglas de correspondencia”. El modelo lógico-matemático utilizado para la sistematización axiomática y, por ende, arquitectónica de dichas teorías fue el empleado por Hilbert en la geometría y por Frege en la aritmética. No obstante, la preocupación exclusiva por los aspectos sintácticosemánticos de las teorías y el desinterés por sus aspectos pragmáticos e históricos hizo que este primer gran proyecto arquitectónico colapsara y se mostrara inoperante para dar respuesta a las nuevas interrogantes que planteaba la naciente concepción historicista en filosofía de la ciencia a partir de la década de los cincuenta, pero sobre todo, en los sesenta del siglo pasado, haciendo que la reflexión metateórica se orientara preferencialmente hacia la praxis científica y no tanto hacia los productos de dicha praxis: las teorías científicas. Sin embargo, del fracaso de este primer gran proyecto arquitectónico en filosofía de la ciencia no se infiere -como en su momento lo advirtieron Suppes y colaboradores- la irrelevancia de los métodos formales de reconstrucción de teorías, pues la matemática y la lógica forman parte de la constitución de las teorías empíricas; y porque del fracaso de un método no se sigue necesariamente el fracaso de todos los posibles métodos, como en su momento fue la propuesta de los historicistas.

Es así como surge el segundo gran proyecto arquitectónico en filosofía de la ciencia: el programa metateórico estructuralista de reconstrucción de teorías. Este programa, a diferencia del anterior, establece un nuevo criterio de identidad para las teorías empíricas al proponer una caracterización modelística y no enunciativista de ellas, cuyas raíces se encuentran en la obra de Suppes y colaboradores inmediatos, y al destacar en la reconstrucción de las teorías no sólo los aspectos sintáctico-semánticos, sino también pragmáticos, sin desconocer, por supuesto, la dimensión diacrónica, i.e., histórica. Si axiomatizar una teoría -como lo enfatizó Suppes (1957) - consiste en definir su contraparte matemática mediante la definición de un predicado teóricoconjuntista de la forma ' $x \in S$ ' el instrumento más adecuado para ello es la matemática, y no la lógica, o la metamatemática. Así, la obtención del predicado de Suppes de una teoría conforma su axiomatización estructural, de tal manera que en esta nueva propuesta arquitectónica 
-que en el estructuralismo resulta más compleja y sofisticada que la de Suppes y sus discípulos- las estructuras que satisfacen el predicado conjuntista son los modelos de la teoría.

El programa estructuralista, sin abandonar el proyecto arquitectónico suppessiano de axiomatización estructural por definición de un predicado teórico-conjuntista, llevo a Sneed a proponer - a comienzos de la década de los setenta- un concepto enriquecido de teoría empírica donde, en contraste con las teorías matemáticas, se destaca su complejidad estructural y, aunque Suppes afronta el problema de la interpretación del formalismo mediante teorías de la medición empíricamente relevantes, en Sneed, de forma explícita, se incorpora en la caracterización de las teorías el domino de sus aplicaciones pretendidas o intencionales. A su vez, la reconstrucción axiomática de las teorías empíricas no se hará mediante la definición de un único predicado teórico-conjuntista, sino a través de una serie de predicados atendiendo a la diversidad de modelos que conforman su componente estructural: actuales, potenciales y potenciales parciales. Como en la versión de Adams de la propuesta de Suppes, la teoría, o mejor, el elemento teórico, consta de dos partes: una puramente formal -la estructura matemática $K$ denominada "núcleo teórico"- y una aplicativa $I$, articuladas en distintos niveles de especificidad (red teórica). Conforme con esta propuesta que, frente a las teorías formales, recupera la complejidad de las teorías empíricas, Sneed propone, como ya fue dicho, la reconstrucción de éstas mediante una serie de predicados teórico-conjuntistas y no de un único predicado como en Suppes, a fin de establecer la función de los distintos modelos del núcleo formal $K$, incorporando en esta empresa arquitectónica nuevas restricciones, además de las leyes, como son las "ligaduras" ("constraints") que -en un contexto holista- permiten establecer conexiones entre los modelos de una teoría, amén de los distintos vínculos interteóricos, de las redes o conexiones entre elementos teóricos mediante relaciones de orden parcial de especialización, etc.

Todo esto nos muestra que el programa estructuralista de reconstrucción de teorías, además de ser un programa realista -como lo atestigua el sinnúmero de teorías exitosamente reconstruidas en poco más de las tres décadas de su existencia- es el que, en la actualidad mejor realiza el ideal arquitectónico kantiano como "el arte del construir 
sistemas". No en vano el libro que de manera sistemática articula y unifica las tesis centrales del enfoque teórico-modelístico estructural y, al mismo tiempo, las ilustra con casos concretos de teorías científicas, lleva por título An Architectonic for Science.

\section{Presuposición analítico-trascendental kantiana y presuposición sneedeana}

La necesidad de superar las dificultades inherentes a la distinción teórico/observacional de los positivistas lógicos y, por ende, de la Concepción Heredada llevó a Sneed a proponer un criterio funcional de teoricidad. Para su formulación, Sneed ve necesario definir -como paso previo- el concepto de "mensurabilidad $T$-dependiente", según el cual una función $f_{i}$ es mensurable de una manera T-dependiente si y sólo si existe un individuo $x$ de $D_{i}\left(x \in D_{i}\right)$ tal que en la exposición existente de la $i$-ésima aplicación de la teoría $T$ no contiene ninguna descripción de un método de medición $f_{i}(x)$ que no presuponga que alguna aplicación de $T$ es exitosa; $f_{i}$ es mensurable de una manera $T$-no dependiente si y sólo si ella no es mensurable de una manera $T$-dependiente (Sneed 1971, p. 31).

En esta definición, Sneed hace uso de las nociones de "teoría física", de "exposición existente de la teoría" y de dos conceptos que, para efectos de la formulación del criterio de teoricidad, son básicos: medición y presuposición. Aunque en la definición no se ve claro el concepto de teoría física que utiliza Sneed -algo que, como veremos, se explicita mejor en la reconstrucción modelo-teórica que de ella hacen W. Balzer y C.U Moulines (Véase Balzer y Moulines 1980, pp. 467494) - no está de más recordar, como ya se dijo, que Sneed no identifica las teorías con una clase o conjunto de enunciados, sino con una clase o conjunto de modelos. Igualmente, con respecto a la expresión "exposición existente de la teoría", hay que señalar que se trata de un concepto pragmático cuya extensión -como sucede con los demás conceptos pragmáticos- no es claramente acotada. Las otras dos nociones son la de "medición" y la de "presuposición", si bien en este escrito me detendré exclusivamente en el concepto de "presuposición" y, en particular, en los posibles vínculos de éste con la noción kantiana de "condición de posibilidad". 
La definición previa del concepto de "mensurabilidad $T$-dependiente" le permite a Sneed introducir la distinción entre conceptos $T$-teóricos y $T$-no teóricos de manera que, de modo general, se puede afirmar que una función $f_{i}$ de una teoría $T$ es teórica con respecto a $T$ syss en cada aplicación de $T$ cualquier método para establecer su valor $\left(\left[f_{i}(x)\right]\right)$ presupone que alguna aplicación de $T$ sea exitosa. Stegmüller, al tratar de clarificar la noción de "presuposición" empleado por Sneed en la anterior formulación, señala que dicha noción "recuerda las notorias dificultades para interpretar correctamente la singular locución de Kant "condición de posibilidad de" (Stegmüller 1983, p. 87), pues cuando Kant en el apartado correspondiente a la Analítica Trascendental de la Crítica de la razón pura y en Principios metafísicos de la ciencia natural (1786) establece que los principios metafísicos que corresponden a las categorías de "sustancia", de "causalidad" y de acción recíproca expresadas a través de juicios sintéticos a priori son condición de posibilidad de la experiencia científica -en particular de la física de Newton- surge el interrogante de establecer si se trata de condiciones de posibilidad necesarias, suficientes o necesarias y suficientes. Si son necesarias, entonces Kant estaría afirmando que su metafísica de la experiencia es una consecuencia de la física newtoniana; pero si son suficientes, entonces estaría expresando que la física newtoniana puede inferirse de su metafísica de la experiencia; y si son necesarias y suficientes, entonces estaría aseverando que su metafísica de la experiencia y la física newtoniana son lógicamente equivalentes. Como es sabido, las tres posibilidades, a la luz de la propuesta kantiana, son igualmente absurdas.

Para resolver esta dificultad Stegmüller propone interpretar la relación de "presuposición" de que se vale Kant en sus argumentos trascendentales como relación de "implicación lógica" entre ciertos enunciados metateóricos, de suerte que las condiciones de posibilidad que el argumento trascendental busca concluir son, en este caso, condiciones necesarias $(C)$ de un enunciado metateórico: "existe una ciencia de la naturaleza" $(E)$ que sería el punto de partida del argumento trascendental, lo que hace que el método de la argumentación trascendental sea regresivo y/o analítico y no progresivo y/o sintético como el de las matemáticas -en particular el de la geometría euclídea-, tal como lo reconoce el mismo Kant en sus Prolegomena (1783) cuando, refiriéndo- 
se a dicho método señala que en él "se parte de lo que se investiga como si fuese dado y se asciende a las condiciones bajo las cuales es solamente posible" (Kant 1971, p. 71).

Lo novedoso de esta interpretación de la noción kantiana de "condición de posibilidad" es la relación que Stegmüller encuentra con la noción de "presuposición" que emplea Sneed en su criterio informal de teoricidad para indicar que la función concreta $f_{i}$, que aparece en la $i$-ésima aplicación $T_{i}$ de la teoría $T$, se mide de manera $T$-dependiente syss existe un $D_{i}$ tal que en cada exposición existente de $T_{i}$ el enunciado que describe el método para hallar el valor $f_{i}\left(x_{0}\right)$, a saber, el enunciado " $S$ " (donde $S$ es la estructura matemática de la teoría $T$ ), implica lógicamente que al menos un enunciado que describe otro método de determinación, a saber, el enunciado " $S$ " es verdadero (is true); i.e., que este enunciado de la correspondiente estructura $S$ satisface las leyes y demás restricciones de $T$. En otras palabras, lo que nos dice el criterio sneedeano de teoricidad es que una función o magnitud $f_{i}$ es $T$-teórica syss todos los métodos involucrados en su determinación son modelos de $T$ o presuponen algunos modelos de $T$.

El criterio informal sneedeano de teoricidad es un criterio fundamentalmente pragmático o funcional -como lo califica Stegmüllerpues tiene que ver con la práctica científica; i.e, con lo que los científicos o las comunidades científicas hacen cuando de asignar valores numéricos a las funciones se trata; sin embargo, tal carácter pragmático o funcional no excluye la posibilidad de darle a dicho criterio una presentación lógica (Balzer 1986, pp. 71-90) o una caracterización modelo-teórica (Balzer y Moulines 1980, pp. 467-494). En este último caso, los autores, luego de advertir que "todos esos criterios intuitivos [se refieren a los de Sneed, Stegmüller y Kamlah] junto con sus aplicaciones a casos concretos son en el fondo insatisfactorios" (ibídem, p. 488 ) y de introducir una definición precisa de lo que ellos denominan "los métodos de determinación" - de los que los métodos de medición usados en el laboratorio son un caso paradigmático-, proponen lo que, para ellos, es una versión (primera versión) del criterio de " $T$ teoricidad". Así: " $f$ es $T$-teórica syss para cualquier conjunto $M_{m}$ : si $M_{m}$ es un método de determinación de $f$ en $T$ entonces $M_{m}$ es un subconjunto de $M(T)$ " (ibídem, p. 473). 
Balzer y Moulines no dudan en reconocer que esta definición (cuya formulación se precisa a lo largo del texto, sobre todo con relación a la problemática afirmación de que $M_{m} \subseteq M(T)$ podría ser interpretada como una traducción modelo-teórica de la explicación que Stegmüller propone de la relación de presuposición en el criterio original de Sneed) cuya idea central, como vimos, es la de que "un método de determinación de [una función magnitud] $f$ presupone una aplicación de $T$ " lo que, para el caso de las funciones $T$-teóricas, significa "que los enunciados que describen el método de determinación im-

plican lógicamente el enunciado [de] que la estructura correspondiente satisface las leyes básicas de T" (ibídem). Esto, en términos puramente modelo-teóricos significa que el conjunto de modelos potenciales de $T\left(M_{p}(T)\right)$ que determinan $f$, i.e., los modelos de determinación de $f$, poseen la misma estructura modelo-teórica que los modelos de $T(M(T))$, si bien, como ya fue advertido, esta situación no siempre se satisface, como es el caso de los métodos de determinación de las funciones $T$-no teóricas donde los modelos de determinación no son modelos potenciales, sino subestructuras de modelos potenciales, i.e., modelos potenciales parciales que resultan de "recortar" en los modelos potenciales las entidades $T$-teóricas. En este caso, los métodos de determinación no dependen de la teoría $T$, sino de teorías disponibles previas a $T$.

Sin detenernos en la discusión de esta reconstrucción modelo-teórica del criterio de teoricidad, conviene destacar que en ella -como en muchas de las interpretaciones del criterio sneedeano de teoricidadse recoge la idea de Stegmüller de asimilar la relación de "presuposición" a la de "consecuencia" o "implicación lógica" entre ciertos enunciados metatéoricos, de suerte que afirmar: " $A$ es el caso presupone que $B$ es el caso" equivale a afirmar: " $B$ se infiere lógicamente de $A$ ".

Aquí, sin embargo, es conveniente observar que, en el caso de la noción de "presuposición" kantiana, no todos los exegetas de Kant comparten la interpretación que Stegmüller propone de la noción de "condición de posibilidad de" en términos de consecuencia lógica de un enunciado metateórico como el enunciado $(E)$ antes mencionado. Strawson, por ejemplo, propone una interpretación de la noción kantiana de "presuposición" alejada por completo de la relación binaria de "implicación lógica" que, en mi criterio, resulta más ajustada a lo 
que Kant denomina "prueba" o "deducción trascendental", y Strawson "argumento trascendental". Con el fin de aclarar esto echaremos mano de la distinción propuesta por Frege entre "implicación lógica" y "presuposición".

Para este autor, la relación de implicación lógica es una relación binaria para la que es válido lo siguiente:

(1) Si " $S_{1}$ implica $S_{2}$ " es verdadero, y " $S_{2}$ " es falso, entonces " $S_{1}$ " es falso (validez del modus tollens).

(2) Si " $S_{1}$ " es falso, entonces " $S_{1}$ implica $S_{2}$ " es verdadero independientemente del valor veritativo de " $S_{2}$ " (implicación vacua o trivialmente verdadera).

La relación de presuposición es una relación binaria para la que es válido lo siguiente:

(1) Si " $S_{1}$ presupone $S_{2}$ " es verdadera y " $S_{2}$ " es falso, entonces " $S_{1}$ " queda indecidida (decimos que "carece de sentido").

(2) Afirmar que " $S_{1}$ presupone $S_{2}$ " nos compromete con la verdad del antecedente " $S_{1}$ "; es decir, aceptamos que $S_{1}$.

Aunado a lo anterior, Frege propone la siguiente definición de "presuposición" que posteriormente será retomada por van Fraassen y por Strawson: $S_{1}$ presupone $S_{2}={ }_{\text {def }} S_{1}$ no es verdadera ni falsa a menos que $S_{2}$ sea verdadera. ${ }^{1}$

Afirmar que " $S_{1}$ no es ni verdadera ni falsa a menos que $S_{2}$ sea verdadera" implica hacer uso de un lenguaje no bivalente, pues si el lenguaje es bivalente la relación resulta trivial, como se desprende de la condición (2) de implicación lógica antes mencionada. Al respecto escribe van Fraassen: "Si $A$ tiene una presuposición que no es verdadera, entonces $A$ no es ni verdadera ni falsa. Así, para que tengamos una relación de presuposición no trivial, un lenguaje no debe ser bivalente" (van Fraassen 1987, p. 174). ${ }^{2}$ Si esto lo aplicamos al caso de

${ }^{1}$ Aunque la palabra "presuposición" fue utilizada por J. Land en 1876 en su trabajo Brentano's Logical Innovations, sin embargo fue Frege quien por primera vez le dio un sentido claro y un soporte teórico sustancial con su teoría semántica y, en particular, con su distinción onto-semántica de sentido/referencia (Cfr. Haack 1974).

${ }^{2}$ No debemos olvidar que la definición fregeana de "presuposición" como algo diferente de la "implicación lógica" tuvo su origen en su célebre discusión con Russell a propósito de los 
Kant habría que reconocer que el enunciado metateórico $(E)$ que hemos mencionado atrás no implica lógicamente el metaenunciado $(C)$ que expresa las condiciones de posibilidad trascendentales de $(E)$, sino que el metaenunciado $(E)$ presupone el metaenunciado $(C)$, de suerte que si $(C)$ es falso, i.e., si las condiciones de posibilidad expresadas por $(C)$ no se cumplen, entonces $(E)$ queda indecidido; i.e., carece de valor de verdad.

Strawson, refiriéndose a lo mismo y con ocasión de la famosa polémica Russell-Frege, expresa:

Decir "El rey de Francia es sabio" es implicar, en algún sentido de "implicar", que hay un rey de Francia. Pero este es un sentido muy especial y extraño de "implicar". "Implicar" en este sentido, no equivale ciertamente a "entrañar" (o implicar lógicamente). Y esto resulta del hecho de que, cuando como respuesta a su enunciado, decimos (como podríamos hacerlo) "No hay ningún rey de Francia", no diríamos ciertamente que estábamos contradiciendo el enunciado de que el rey de Francia es sabio. No estamos diciendo, por cierto, que es falso. Estamos más bien dando razón para decir que la cuestión de si es verdadero o falso no se plantea (Strawson 1995, p. 67).

De forma similar, si adoptamos esta noción de "presuposición" como algo distinto de la "implicación lógica" y la aplicamos al criterio de teoricidad de Sneed podemos decir que una función o magnitud concreta $f_{i}$ que aparece en la $i$-ésima aplicación $T_{i}$ de la teoría $T$ se mide de manera $T$-dependiente syss existe un $D_{i} \cap D_{I}\left(f_{i}\right)$ tal que en cada manifestación existente de $T_{i}$ el enunciado que describe el método para hallar el valor $f_{i}\left(x_{0}\right)$, a saber, el enunciado " $S$ " -donde $S$ es la estructura matemática de la teoría $T$ - presupone, como condición de posibilidad necesaria, que otro enunciado que describe otro método de medición, a saber, el enunciado " $c_{j} \in S$ " es verdadero. De acuerdo con esta modificación que atiende a la definición fregeana de "presuposición", podemos decir que en caso de que el enunciado $S^{\prime}$ "fue-

nombres propios. Si bien ambos están de acuerdo en la equivalencia entre el significado de los nombres propios y de las descripciones definidas, sus explicaciones respecto del modo en que éstas funcionan es diferente. Mientras para Russell una descripción definida como "El actual Rey de Francia es calvo" implica —entre otras cosas- la proposición: "Existe una persona que es en la actualidad Rey de Francia", pues que lo hay es parte de lo que dice, para Frege, la proposición: "El actual Rey de Francia es calvo" presupone la proposición: "Hay un actual Rey de Francia”, y aquella no es ni verdadera ni falsa a no ser que estas última sea verdadera. 
se falso, entonces el enunciado $S$ " no sería falso -como sucedería si aplicásemos el modus tollens - sino que su valor de verdad quedaría indecidido, o en palabras de Frege, carecería de referencia ("Bedeutung") y, en consecuencia, no habría razón para afirmar (o para negar) que la función concreta $f_{i}$ que aparece en la $i$-ésima aplicación $T_{i}$ de la teoría $T$ es $T$-teórica. En todo caso, lo que hay destacar es que, cuando se trata del valor de una determinada función, se asume que el proceso de medición por medio del cual se obtiene este valor está gobernado por una teoría $T$, o como lo acabamos de señalar, presupone una teoría $T$, de suerte que dicho modelo de medición y/o determinación es -al menos para el caso de las funciones $T$-teóricas- un subconjunto de los modelos actuales de T. Si en todos los casos los métodos de medición y/o determinación de una determinada función o magnitud son modelos actuales de la teoría, es decir, son modelos gobernados por $T$ en el sentido de ser $T$-dependientes, entonces la función es $T$-teórica; si no dependen de $T$ sino de una teoría $T^{*}$ diferente de $T$, entonces la función sería $T$-no teórica. Pero si no dependen de $T$ ni de otra teoría $T^{*}$ diferente de $T$, i.e., si no existe ningún método de determinación para la función, entonces afirmar que ella es $T$-teórica o $T$-no teórica carece de sentido.

Finalmente, cuando hablábamos de las condiciones que debe cumplir la relación de "presuposición" señalábamos como condición (2) lo siguiente: " $S_{1}$ presupone $S_{2}$ " nos compromete con la verdad del antecedente " $S_{1}$ ", es decir, aceptamos que $S_{1}$. Esto, en el caso del criterio de teoricidad de Sneed, tiene que ver con su afirmación de que para que una función o magnitud sea $T$-teórica se requiere, como condición suficiente y necesaria, que todos los métodos de medición aceptados presupongan la teoría $T$, es decir, sean modelos de la teoría $T$. El hecho de introducir la expresión "aceptados" para calificar dichos métodos o procedimientos de medición hace que esta condición tenga un carácter pragmático, pues tal aceptación depende de la decisión de quienes integran una determinada comunidad científica. En este caso, Kant podría decir -como lo afirma la primera premisa de sus argumentos trascendentales que, en su mayoría, son argumentos antiescépticos- que se parte de algo dado cuya existencia es una prueba de su posibilidad -como posibilidad fáctica. Sneed, de manera análoga, parte de la aceptación de la comunidad científica de unos proce- 
dimientos de medición y/o determinación cuya existencia es -como diría Kant- una prueba de su posibilidad, de tal modo que lo que hay que preguntar, como de algún modo lo hace Sneed, no es si las funciones $T$-teóricas y $T$-no teóricas son posibles, sino cómo son posibles, i.e., qué es lo que ellas presuponen. Con base en esto se podrá establecer si la función o magnitud en cuestión es $T$-teórica, $T$-no teórica o simplemente si carece de sentido afirmar una u otra cosa.

\section{El a priori kantiano y el a priori estructuralista}

Intentar plantear algún tipo de analogía entre el a priori kantiano y el a priori sneedeano parecería un poco descabellado toda vez que para el primero las dos características que lo definen son la necesidad y la estricta universalidad, y esto, hoy día, está alejado de cualquier consideración de la ciencia, incluyendo por supuesto, la sneedeana.

Para el pensador königsberguense la distinción a priori/a posteriori es epistémica, pues se refiere a la independencia o no del conocimiento de la experiencia, respectivamente. Para él, el sistema conceptual básico del entendimiento humano, a saber, el sistema de los conceptos puros del entendimiento o categorías, es un sistema a priori y, sus componentes, al igual que el de los principios que de ellos se derivan, poseen un carácter de necesidad y de estricta universalidad y, como tales no están sujetos a ningún cambio potencial, vale decir, son intemporales.

Ahora bien, aunque Kant propone las categorías y los principios del entendimiento como condiciones necesarias (a priori) de la experiencia y/o conocimiento objetivo, dichas condiciones no son, como él pretendía, condiciones absolutas, pues sólo dicen relación a una clase de experiencia y/o conocimiento, es decir, el conocimiento científico de su época. Su error consistió entonces en convertir las supuestas condiciones metafísicas de posibilidad del conocimiento de su época en condiciones absolutas de posibilidad para el conocimiento objetivo en cualquier época. Sobre la base de reconocer este error, muchos de sus discípulos y seguidores vieron la necesidad de liberalizar y relativizar dichas condiciones de posibilidad, asignándoles para su validez un marcador temporal, pero esta modificación no tuvo el impacto deseado en el ámbito de la filosofía de la ciencia, pues buena parte de ella 
tiene su origen en el empirismo que, como es sabido, es ajeno a cualquier tipo de apriorismo, incluyendo el relativizado de algunos de los epígonos de Kant; para la concepción empirista, todas las hipótesis de las teorías empíricas deben pasar por el tribunal de la experiencia y ninguna de ellas puede ser inmune a este control. Pero ni siquiera la posición kuhniana acerca del desarrollo de la ciencia pareciera justificar un acercamiento con la concepción kantiana liberalizada, pues si se restringe su vago concepto de "paradigma" al conjunto de sus ejemplares paradigmáticos, tal componente no es a priori, sino empírico, por ello, se hace necesario tomar en consideración únicamente el primer componente de lo que para Sneed es una teoría empírica, es decir, el núcleo formal $K$, dejando de lado el segundo componente, i.e., el componente de las aplicaciones intencionales $I$ y su subconjunto de aplicaciones paradigmáticas $I_{0}$. El núcleo $K$ es -en palabras de Stegmüller (1973) - "el componente a priori de una teoría" en el sentido de que "hasta tanto las personas dispongan de esa teoría, este componente permanece invariable, es inmune a las tormentas del mundo de la experiencia y no necesita ser inmunizada por medio de "refinamientos convencionalistas" (ibídem, p. 309). Por supuesto, en el caso del estructuralismo, en contraste con Kant, no se trata de un a priori absoluto, sino de uno relativizado, pues un núcleo estructural formal, por más refinado y perfeccionado que esté, y por muchas que hayan sido sus aplicaciones exitosas, nunca estará exento de entrar en contradicción con la experiencia o, como en el caso de las revoluciones científicas, de entrar en un posible conflicto con un nuevo núcleo $a$ priori que resuelva todos los problemas que éste resolvía, y también algunos otros que no era capaz de resolver (las anomalías). Pensar lo contrario es convertir las teorías científicas en dogmas.

De conformidad con la noción de "red teórica" introducida por el estructuralismo, una "teoría" es algo que se desarrolla en el tiempo conservando lo que se ha denominado una clase de "identidad genidéntica", lo que nos permite hablar de "evolución teórica", tanto en el sentido de "evolución normal" como de "evolución no normal". La analogía formal entre el componente a priori relativo de Kant y el componente a priori de una teoría de Sneed tiene que ver con la evolución normal de ésta pues, a pesar de ella, es decir, de la existencia de nuevos elementos teóricos en los que se introducen nuevas leyes o se 
abandonan leyes ya admitidas, se introducen o abandonan condiciones de ligadura, etc., el núcleo estructural formal de la teoría permanece y, en ese sentido, el núcleo estructural $[\mathbf{K}]$ es a priori. En este punto es conveniente advertir que esta "evolución normal" no se corresponde exactamente con lo que Kuhn denomina el "desarrollo" o la "dinámica de la ciencia normal", pues en este último caso se introducen aspectos que no atañen directamente a lo que los estructuralistas denominan "evolución normal": observación de nuevos fenómenos, descubrimiento de datos que confirmen o rechacen leyes y condiciones de ligadura especiales admitidas en forma hipotética, etc. Todo esto hace parte de la vaga noción de "paradigma" introducida por Kuhn.

No podemos pasar por alto que una de las características del "componente a priori de una teoría” -como Stegmüller (1973) se refiere a su núcleo formal $K$ - es su esencial irrefutabilidad por la experiencia, que llevó a Moulines (1982) a precisar el componente de las generaciones simbólicas como uno de los componentes esenciales del paradigma o de lo que Kuhn, algunos años después y sin mucho éxito denominó: "matriz disciplinar". En el decir de Stegmüller, las llamadas "generalizaciones simbólicas" de Kuhn no son otra cosa que las leyes fundamentales del llamado "núcleo estructural $K$ " de la teoría. Sin embargo, como es fácil colegirlo de la lectura de la Posdata (1969) a La estructura de las revoluciones científicas, dichas generalizaciones simbólicas, más que ser enunciados que expresan leyes son esquemas de generalización o, como diría Frege, "formas proposicionales" -Kuhn habla de "formas simbólicas"- susceptibles de recibir diversas interpretaciones. Sobre la base de este reconocimiento, Moulines concibe las generalizaciones simbólicas de Kuhn como principios-guía, pues dicha denominación -según su opinión- refleja mejor la función heurística que dichas entidades cumplen en la ciencia. ${ }^{3}$ Su propuesta la ejemplifica con dos casos paradigmáticos: la ley fundamental de la mecánica clásica y la ley fundamental de la termodinámica fenomenológica; luego de realizar una reconstrucción lógica de cada una de esas leyes y de advertir algunas interesantes consecuencias teóricas y metodológicas,

${ }^{3}$ La expresión "principio guía" -además de ser algo que ya existía en la literatura metodológica- recuerda la idea que E. Nagel tenía de los axiomas de una teoría, para quien antes que ser afirmaciones acerca de las cuales se producen determinados fenómenos, son guías o reglas metodológicas que orientan al científico en la búsqueda de parámetros adecuados cuando de explicar el comportamiento de algo se trata ( $c f r$. Nagel 1961, cap. VII). 
Moulines muestra que ambas leyes, dada su forma lógica, son irrefutables por la experiencia, esto con la salvedad de que se trata de una "irrefutabilidad de segundo orden", para mayor escándalo de los falsacionistas popperianos y de los inductivistas carnapianos. Pero este hecho no convierte dichas leyes en definiciones postulacionales como cuando Kuhn (1970) dice que " $f=m \cdot a$ o $I=V / R$ funcionan en parte como leyes, pero también en parte como definiciones de algunos de los símbolos que muestran" (p. 281), pues ellos no establecen reglas lingüísticas sobre el uso de los términos. Pero a pesar de no ser definiciones y, por ende, tautologías, Moulines señala que es tal su estructura que - en la terminología de Sneed-, cualquier modelo parcial (no teórico) podría ser "extendido" o "completado" trivialmente hasta transformarse en un modelo completo (teórico) tanto de la mecánica clásica, como de la termodinámica fenomenológica en el sentido de satisfacer las leyes fundamentales de estas teorías. Siendo así, las leyes fundamentales de estas dos teorías -y posiblemente de muchas, si no de todas- son empíricamente vacuas por no establecer ninguna restricción de contenido empírico. Sin embargo, el mismo Moulines advierte que hablar de "empíricamente vacuo" causaría confusión, pues serían enunciados analíticos, de suerte que en vez de la expresión "vacuamente verdaderos" propone la expresión "empíricamente irrestrictos", es decir, completamente irrefutables por la experiencia. Esta caracterización, además de precisar la inmunidad del paradigma kuhniano, nuevamente permite establecer una analogía entre el a prio$r i$ de Kant -una de cuyas marcas distintivas es la de ser irrefutable por la experiencia- y las leyes fundamentales de las teorías como elemento indispensable para hablar de modelos actuales en el núcleo de la teoría en la concepción estructuralista. Siguiendo a Kant diríamos que se trata de un a priori no puro como, según este autor, sucede con el juicio sintético a priori: "todo cambio tienen una causa", pues aunque es, valga la redundancia, un a priori, no es uno puro, es decir, absolutamente independiente de toda experiencia ya que el concepto de "cambio" se obtiene a partir de la experiencia.

Adicionalmente hay que decir que existe una analogía estrecha entre la función de los principios últimos ("Grundsätze") a los que Kant se refiere en el acápite de la Analogía de la Experiencia en la Crítica de la Razón Pura y los principios-guía de que hemos venido hablan- 
do. En el primer caso, los principios metafísicos últimos son guías $o$ reglas metodológicas - en el sentido de metodología trascendentalpara el descubrimiento de leyes más especiales, de suerte que poseen, como dice Kant, "una valor meramente regulador y no constitutivo" (Kant 1928, p. 211; A 180 / B 223). Así, los principios metafísicos de la permanencia o sustancia (primera analogía), de la sucesión o causalidad (segunda analogía) y de la simultaneidad o acción recíproca (tercera analogía) sirven de regla para el descubrimiento de leyes como la de conservación del momento, la segunda ley de Newton y la de acción reacción -tercera ley de Newton- en la mecánica clásica. Teniendo esto, podemos afirmar que los principios metafísicos últimos de que nos habla Kant, al igual que los principios-guía de que nos habla Moulines, cumplen, antes que nada, una función heurística. En el primer caso, por ejemplo, las leyes de la física clásica -como lo reconoce el filósofo königsberguense- no son más que sus ejemplificaciones en el dominio de la experiencia empírica de aquellos principios últimos (“Grundsätze”), en el segundo, los principios-guía de la mecánica clásica y de la termodinámica fenomenológica no son ni meras descripciones de hechos particulares ni de un conjunto de hechos particulares ni definiciones de términos como "fuerza", "entropía”, "energía interna”, etc., como lo sugiere Kuhn, ni de situaciones que a veces son descripciones y a veces definiciones como lo proponen algunas posiciones escépticas, sino "matrices conceptuales a partir de las cuales se pueden derivar - pero no en el sentido de una deducción lógica- importantes leyes empíricas sobre la naturaleza" (Moulines 1982, p. 105).

Los principios-guía cumplen una función análoga a la del paradigma kuhniano, al ser más que una promesa de éxito una realización palpable, pues ellos, en tanto principios-guía, son reglas que nos orientan en la identificación de las funciones o funcionales, de sus parámetros apropiados y de sus relaciones funcionales para la formulación de leyes especiales -cada vez más especiales- en campos de aplicación cada vez más acotados. Como se puede ver, ellos comparten, con los principios metafísicos últimos ("Grundsätze") de que nos habla Kant, su función heurística y, como estos, son irrefutables por la experiencia. Claro está que en el caso de los principios-guía estamos ante principios "empíricamente irrestrictos", de suerte que no son completamente independientes de la experiencia, pues de serlo serían completamente 
vacíos de contenido empírico. Ellos son por completo irrefutables e inverificables por la experiencia, causando que sobre ellos -como sobre los principios metafísicos de Kant- no se puede establecer ni siquiera sus grados de corroboración, confirmación, likelihood, etc.; son, por decirlo de algún modo, principios que se mueven entre el a priori y el a posteriori kantiano, quizás por eso sería mejor llamarlos "a priori no puros".

\section{Realismo y antirrealismo en las filosofía kantiana y estructuralista}

Pretender establecer algún tipo de analogía entre las concepciones kantiana y estructuralista con respecto a los problemas semánticos de la referencia y del sentido tanto de los términos científicos como de las teorías no deja de ser problemático, toda vez que se trata de propuestas filosóficas con propósitos y desarrollos metodológicos distintos y, sobre todo, porque en el caso del estructuralismo no se posible hablar de un punto de vista uniforme y unificado, máxime porque este tipo de problemas metateóricos no han sido de interés explícito -al menos desde el punto de vista de la filosofía general de la ciencia- por parte de quienes se han ocupado de identificar teorías con la metodología estructuralista. Es quizás Moulines quien, haciendo uso de la semántica Frege y de la concepción ontológica de Quine, se ha ocupado con mayor empeño de este tipo de problemas y, en particular, de la constitución de esa nueva disciplina que él denominó "onto-epistemosemántica”, coloreada con el matiz wittgensteniano de la pragmática por aquello de que "el significado de una palabra es su uso en el lenguaje" (Wittgenstein 1988, p. 61). Esto explica por qué para el desarrollo de este numeral haremos uso preponderante de los planteamientos de Moulines sobre estos asuntos.

Kant defiende dos doctrinas claramente diferenciables en relación con los problemas de la realidad del mundo exterior y su conocimiento: el realismo empírico y el idealismo trascendental, que en algunos casos, reviste la forma de un fenomenalismo de tipo berkeleyano.

Su realismo empírico es un realismo ontológico que aunque guarda semejanza con el "realismo exterior" de Strawson y con el "realismo referencial" de Moulines, no se identifica con ellos, como tendré oportunidad de mostrarlo más adelante. El realismo empírico kantiano pre- 
supone que las cosas, el universo, están "ahí” y que ese "estar ahí" (“ $d a$ sein") de las cosas, es independiente de que sean percibidas o concebidas, o para usar un lenguaje más cercano al filósofo königsberguense, "están ahí" con independencia de las condiciones que hacen posible su percepción y su conocimiento, a saber, las intuiciones puras de espacio y tiempo, y las categorías y principios, respectivamente. A esta cosas Kant las denomina noumena y, en ocasiones, les atribuye "poderes" causales, lo que, desde el punto de vista de su posición crítica, constituye una caída en el dogmatismo. A este realismo empírico (ontológico) le corresponde un idealismo trascendental que, en mi opinión, es una forma de antirrealismo epistemológico, pues considera que las cosas que están "ahí”, los noumena, son incognoscibles; tales cosas que están "ahí” y que Kant, a su vez, denomina "cosas en sí ("an sich”), no nos son cognoscibles - al menos a nosotros los humanos- pues, de conformidad con su propuesta epistémica, nuestro conocimiento está limitado y circunscrito a lo que el mismo Kant denomina "la experiencia posible"; este antirrealismo epistemológico convierte el realismo empírico (ontológico) en un tipo de realismo ingenuo, pues a pesar de que Kant asume un compromiso ontológico respecto a la existencia de las cosas "ahí fuera" como algo que se da con independencia de nuestros marcos preceptuales y cognitivos, sin embargo, al mismo tiempo plantea que no podemos presuponer -como presuposición epistemológica- que a ellas se refieran nuestros conceptos, y menos aún, saber que esas cosas "ahí fuera", los noumena, realmente existen, pues de la categoría modal de "existencia" - al igual que las de "posibilidad" y de "necesidad"sólo existe un uso legítimo de dicto y no de re.

A diferencia del realismo ontológico o externo y del antirrealismo epistemológico de Kant, el llamado "realismo científico" -del que Popper y Bunge son dos de sus más notables representantes- implica los siguientes presupuestos: a) Las cosas "ahí fuera" (el universo) existen de manera autónoma, con independencia de los marcos conceptuales llamados "teorías científicas"; b) Dichas cosas poseen un grado de organización, están "ahí" de una y sólo una manera (estructura de la realidad); c) El conocimiento científico nos brinda una representación verdadera o aproximadamente verdadera de esas cosas. Como es fácil advertirlo, las primeras dos presuposiciones son ontológicas y la tercera es epistemológica, sin embargo, al hablar de "representación verda- 
dera o aproximadamente verdadera" se compromete con una forma peculiar de realismo epistemológico que Moulines denomina "realismo alético", "porque la noción de verdad es la que está en juego en el fondo" (Moulines 1991, p. 132).

Como veremos a continuación, la concepción estructuralista y, de modo especial, la concepción de Moulines, propone un tipo de reflexión onto-epistemo-semántica de las teorías científicas cuyos presupuestos ontológicos y epistemológicos difieren de los de Kant y de los llamados "realistas científicos".

Moulines -como en su momento lo había hecho Sneed (1983)circunscribe su reflexión a las teorías científicas, pues sin desconocer la existencia de otras formas de saber y de otros tipos de lenguaje, es en estas teorías donde las cuestiones ontológicas y epistemológicas revisten mayor interés. Para este autor, el realismo empírico kantiano es una forma de "realismo puramente ontológico" y, como tal, constituye la forma más débil del llamado "realismo referencial", pues aunque postula la existencia de cosas "ahí fuera" (los noumena), con independencia de nuestros marcos preceptuales (espacio y tiempo) y cognitivos (las categorías), al mismo tiempo propone la imposibilidad de su conocimiento y, en consecuencia, la imposibilidad de establecer si dichas cosas realmente existen. Una versión fuerte de este realismo referencial añadiría al compromiso ontológico un compromiso epistemológico, de manera que el realismo ontológico pasaría a convertirse en un realismo también epistemológico. Sin embargo, a partir de un detenido análisis de la tesis de la inconmensurabilidad, propuesta por Kuhn y Feyerabend, del principio de la inescrutabilidad de la referencia, postulado por Quine, de la teoría causal de la referencia de Kripke y de la teoría convergentista de la verdad, propuesta por primera vez por Peirce y retomada por Popper, Moulines llega a la conclusión de que el realismo epistemológico, tal como lo formula el llamado "realismo científico", es insostenible. Sin pretender ahondar en los distintos argumentos de que se vale Moulines para criticar el realismo epistemológico, pues de ello me he ocupado en otro lugar (Jaramillo 2001), veámos cuál podría ser, desde el punto de vista de la concepción estructuralista y más específicamente desde la propuesta onto-epistemo-semántica de Moulines, la respuesta adecuada a los problemas de la referencia y del sentido tanto de los términos científicos como de las teorías de ciencia, 
y cuál sería la posición frente al debate realismo/antirrealismo en los niveles ontológico y epistemológico.

Para comenzar es necesario hacer mención de la discusión de Sneed (1983) sobre el realismo científico. Para él, el compromiso ontológico básico de este realismo es que todos los individuos y propiedades que aparecen en un determinado contexto científico poseen el mismo estatus ontológico, lo que, en opinión de Moulines (1991), significa -como tesis semántica básica- que todos los términos individuales y relacionales de las teorías científicas vienen determinados de la misma manera.

Para entender qué significa la afirmación anterior se hace necesario echar mano de la distinción semántica fregeana entre sentido ("Sinn") y referencia ("Bedeutung"), y con base en el principio ontosemántico fundamental de Quine (1953), según el cual "ser es ser el valor de una variable ligada", proponer -como lo hace Moulines- una interpretación de dicha dicotomía en relación con los términos cuantitativos (magnitudes o funciones) de aquellas teorías que hacen uso de este vocabulario.

Para Frege, la referencia ("Bedeutung") de una unidad semántica -llámese "nombre propio", "término conceptual" o "proposición"- es lo designado por dicha unidad semántica y el sentido ("Sinn") el modo o manera como dicha unidad semántica designa su referencia; si bien es preciso advertir que para este autor la noción de "referencia" no se identifica con la de "objeto", pues para él existen dos clases de referencias, los objetos y las funciones, entendiendo por los primeros entidades completas y saturadas, y por los segundos entidades incompletas o insaturadas ( $c f r$. Frege 1973, p. 34).

Si nos detenemos en las teorías científicas cuantitativas tenemos que decir que desde una interpretación instrumental de la semántica de Frege -como la que propone el operacionalismo-, la referencia de las funciones o magnitudes que conforman el vocabulario básico de dichas teorías es una clase de dupla consistente en objetos empíricos y números, y el sentido, el modo o manera como establecemos el valor numérico de dichas funciones, i.e., la clase de prácticas o técnicas empíricas de laboratorio o con lápiz y papel. Para los operacionalistas -como Bridgman, por ejemplo- el sentido de los términos cuantitativos o magnitudes se reduce a estas prácticas o técnicas, pues ellas son la forma como podemos establecer su referencia, i.e., su valor numérico. 
Aunque esta propuesta parece bastante razonable, pues es lo que suele hacerse cuando se quiere establecer el valor numérico que corresponde a ciertos objetos empíricos según una determinada función, se trata sin embargo de una idea demasiado simplista y causante de confusión sobre el sentido de los términos cuantitativos, pues la medición va más allá del mero uso o empleo de aparatos para la misma, ya que éstos -también denominados sistemas por Suppes (1962)- son modelos o realizaciones posibles de los datos cuya estructura "satisface" las leyes o axiomas de una teoría en un sentido análogo, aunque no idéntico, al de Tarski. Decimos esto pues en realidad son conjuntos de subestructuras parciales que para ser modelos de la teoría en el sentido de Tarski deben ser ampliados con funciones $T$-teóricas, de modo que se obtengan modelos que satisfagan los axiomas y demás restricciones de la teoría. Esta es la razón por la que Suppes y los estructuralistas cuando se refieren a dichos sistemas los denominan "modelos de datos" o "modelos de determinación" y no "modelos" sin más. Pero lo interesante es que éstos, de los cuales nos valemos para determinar la extensión o referencia de los términos teóricos, no se dan aislados, lo que hace que Moulines proponga un primer sentido holista de las teorías científicas de cara a los procedimientos operacionales de determinación que conlleva una superación y enriquecimiento de la semántica operacionalista de Bridgman, de Giles y de algunos miembros del Círculo de Viena. En este caso, las condiciones de interconexión entre los distintos modelos de una misma teoría -incluyendo por supuesto las realizaciones posibles- son las llamadas "condiciones de ligadura" ("contraints") que, a diferencia de las leyes o axiomas de la teoría, no operan a nivel de modelos aislados sino de grupos de modelos, razón por la cual también se les conoce como "restricciones cruzadas". Esto, como ya fue advertido, permite pensar en un primer principio holista de las teorías que podría llamarse "Principio de conexión intramodélico", PCI.

Pero aunque se trata de un principio holista en el sentido de que el todo tiene primacía sobre las partes, pues lo que importa no son los sistemas o realizaciones posibles de datos aisladas sino sus grupos o totalidades, es necesario también tener en cuenta que la determinación del sentido de un término cuantitativo no depende únicamente de los sistemas o realizaciones posibles interconectados, sino de las re- 
laciones de ese término con otros básicos en una determinada teoría que puede ser la misma para el caso de las funciones $T$-teóricas, con el problema que ello conlleva, u otra distinta para el de las funciones $T$-no teóricas. No obstante, como lo recuerda Moulines, esto no significa "que el significado de un término científico viene determinado por todo el complejo conceptual, es decir, por toda teoría en la que aparezca dicho término" (Moulines 1991, p. 195), como de algún modo lo propone el holismo radical de Quine e incluso el de Davidson. A este nuevo principio holista moderado lo podríamos llamar "Principio de Interconexión Conceptual", PIC. Finalmente, hay que señalar que en la mayoría de las teorías las conexiones no se establecen únicamente entre los términos de la misma teoría -como es el caso de los T-teóricos- sino con complejos conceptuales de otras teorías, de suerte que para determinar el significado de una de éstas no sólo hay que presuponer únicamente por los principios holistas de conexión entre los sistemas o realizaciones posibles ( $\mathrm{PCI}$ ), junto con el principio de interconexión conceptual (PIC), sino, además, otro principio que podríamos denominar "Principio de Interrelación Teórico (PIT).

Estos tres principios hacen que la semántica operacionalista de Moulines no sólo supere el instrumentalismo de los operacionalistas clásicos (Bridgman, por ejemplo), sino que su calificativo de "holismo moderado" pone de presente que las totalidades a que hace referencia cada uno de los principios antes mencionados sean, en cada caso, totalidades muy delimitadas, y bajo ningún concepto, aspiran a cubrir la totalidad de una disciplina, menos aún, la totalidad de la ciencia, de suerte que podría calificarse, si así se quiere hablar, de "holismo local".

El apoyo metodológico que Moulines brinda a la semántica operacionalista mediante el recurso a la teoría fregeana del significado en general no sólo permite esclarecer el modo de determinación del sentido y referencia de los términos científicos y en particular de los términos cuantitativos, sino, además, establecer la clase de referencia de la teoría, también conocida como "ontología de la teoría". En este caso, los modelos datos o de determinación -con los que la teoría asume un compromiso ontológico- son subestructuras empíricas que mediante la adición de funciones $T$-teóricas pueden devenir modelos de la teoría si satisfacen las leyes y conjuntamente las condiciones de ligadura, lo que significa la subsunción de la experiencia por parte de 
la teoría. Esto, por supuesto, no significa que dicha experiencia pueda, a su vez, ser subsumida por otra teoría con la que la anterior tiene una relación de aproximación interteórica, de tal modo que la primera queda ontológicamente reducida a esta otra teoría.

Lo anterior nos permite concluir que para la concepción estructuralista, y en particular para Moulines, dicho compromiso ontológico implica asumir una forma de realismo ontológico que si bien reconoce la existencia de las cosas como algo que existe "ahí", lo que, de suyo, constituye un buen presupuesto, tal compromiso no implica -como en el caso del realismo científico- que ellas estén estructuradas de una y sólo una manera (la estructura de la realidad), pues presuponerlo significa ser víctima de lo que en su momento Stegmüller cuestionó como "una de las ficciones más explícitas y tácitamente más caras a muchos filósofos" (Stegmüller 1979, p. 29), la de creer que "la realidad posee un inventario dado previamente a todo lenguaje, que en parte consiste en hechos, en parte en estado de cosas ciertamente posibles pero no realizados" (ibídem). Para Stegmüller, "la constitución de la realidad en estados de cosas y hechos es relativa no a una consciencia pensante, a un sujeto trascendental -haciendo clara alusión a Kant- , sino al lenguaje que describa esa realidad" (ibídem, p. 30). El hecho de hablar de la "constitución de la realidad" enfatiza que la realidad de lo real (no lo real en sí) dice relación a "algo" que, en tanto constructo, depende del lenguaje, o para ser más precisos, de los marcos conceptuales que llamamos teorías, y no de "algo" independiente de ellos.

En síntesis, esta particular propuesta de algunos estructuralistas defiende un tipo de realismo ontológico y al tiempo un tipo de antirrealismo epistemológico o de instrumentalismo de las teorías, entendidas éstas dentro de la visión de holismo moderado o local y de holismo radical a la manera de Quine. La misma noción de "interpretación" que propone Moulines (1982) para caracterizar tanto el trabajo de la filosofía de la ciencia, como de las teorías científicas, contrasta significativamente con la que el realismo científico propone del científico en sí como descubridor. Descubrir no es en realidad un acto creador y nada más ajeno a este acto demiúrgico del hombre de ciencia que la imagen del científico como descubridor propia del realismo científico, y en particular, de su realismo alético. 


\section{Referencias}

Balzer, W., 1986, "Theoretical Terms: A New Perspective", The Journal of Philosophy, vol. 33, no. 2, pp. 71-90.

Balzer, W. y C.U. Moulines, 1980, “On Theoreticity”, Synthese, vol. 3, no. 44, pp. 467-494.

Balzer, W., C.U. Moulines y J.D. Sneed, 1987, An Architectonic for Science. The Structuralist Program, D. Reidel, Dordrecht.

Bourbaki, N., 1968, Theory of Sets, Hermann-Addison Wesley, París/Nueva York.

—, 1891, Funktion und Begriff, Hermann Pohle, Jena.

Haack, S., 1974, Deviant Logic, Cambridge University Press, Cambridge.

Jaramillo, J.M., 2001, ¿ Es la ciencia una rama de la literatura fantástica? Pretexto para una reflexión sobre el realismo, Universidad de Caldas, Manizales.

Kant, I., 1781/1787, Kritik der Reinen Vernunft, Johann Friederich Hartknock, Riga.

— Word Auftreten Könen, Johann Friederich Hartknock, Riga. Traducción castellana: Prolegomenos, Aguilar, Buenos Aires.

Kuhn, T.S., 1962/1970, The Structure of Scientific Revolutions, University of Chicago, Chicago.

Moulines, C.U., 2006, La Philosophie des Sciences. Linvention d'une Discipline. (Fin XIXe-debut XXIe siècle), Rue D’Ulm/École normale supérieure, París.

Nagel, E.,1961, The Structure of Science, Harcourt, Nueva York.

Quine, W.V.O., 1953, From a Logical Point of View, Harvard University, Cambridge.

Rescher, N., 1979, Cognitive Systematization, Basil Blackwell, Oxford.

Sneed, J.D., 1971, The Logical Structure of Mathematical Physics, Reidel, Dordrecht.

— 1983 , "Structuralism and Scientific Realism”, Erkenntnis, vol. 19, pp. 345370 .

Stegmüller, W., 1973, Theoriensitrukturen und Theoriendynamik, Springer, Heildelberg.

Strawson, P., 1950, “On Refering." Mind, vol. 59, no. 235, pp. 320-344. Versión castellana de L. Valdés Villanueva (comp.) en La búsqueda del significado. Lecturas de filosofía del lenguaje, Tecnos, Madrid.

Suppes, P., 1957,Introduction to Logic, D. van Nostrand, Nueva York.

— 1962, "Models of Data", en Suppes y Tarski 1962, pp. 252-261.

— 1969, Studies in the Methodological and Foundations of Science, Reidel, Dordrecht.

Suppes, P. and A. Tarski, 1962, Logic Methodology and Philosophy of Sciences, Stanford University, Stanford. 
JUAN MANUEL JARAMILLO

Wittgenstein, L., 1953, Philosophische Untersuchungen, Blackwell, Oxford.

Van Fraasen, B.C., 1971, Formal Semantic and Logic, Macmillan, Nueva York.

Recibido el 8 de septiembre de 2011

Aceptado el 30 de noviembre de 2011 